\title{
Technical note: Validation of sensor-recorded lying bouts in lactating dairy cows using a 2-sensor approach
}

\author{
A. Kok, ${ }^{*}{ }^{1}$ A. T. M. van Knegsel, $†$ C. E. van Middelaar, ${ }^{*}$ H. Hogeveen, $\neq$ B. Kemp, $\dagger$ and I. J. M. de Boer ${ }^{*}$ \\ ${ }^{*}$ Animal Production Systems group, Department of Animal Sciences, Wageningen University, PO Box 338, 6700 AH Wageningen, the Netherlands \\ †Adaptation Physiology group, Department of Animal Sciences, Wageningen University, PO Box 338, 6700 AH Wageningen, the Netherlands \\ ‡Business Economics group, Wageningen University, PO Box 8130, 6700 EW Wageningen, the Netherlands
}

\section{ABSTRACT}

Lying behavior is a relevant indicator for the evaluation of cow welfare. Lying can be recorded automatically by data loggers attached to one of the hind legs of a cow. A threshold for the duration of a lying bout (LB) record is required, however, to discard false records caused by horizontal leg movements, such as scratching. Previously determined thresholds for similar sensors ranged from $25 \mathrm{~s}$ to $4 \mathrm{~min}$. We aimed to validate LB recorded by the IceQube sensor (with IceManager software; IceRobotics, South Queensferry, UK) and to determine a threshold to distinguish true from false LB records in lactating dairy cows. A novel method of validation, which does not require time-consuming behavioral observations, was used to generate a larger data set with potentially more incidental short LB records. Both hind legs of 28 lactating dairy cows were equipped with an IceQube sensor for a period of 6 $\mathrm{d}$ and used as each other's validation. Classification of $\mathrm{LB}$ records as true (actual LB) or false (recorded while standing) was based on 3 assumptions. First, all standing records (absence of LB records) were assumed to occur while standing. Second, false LB records due to short leg movements could not occur in both hind legs simultaneously. Third, true LB only occurred if the LB records of the paired sensors coincided. False LB records constituted $4 \%$ of the records. Based on a maximum accuracy of 0.99 , a minimum duration of LB records of $33 \mathrm{~s}$ was determined, implying a sensitivity of 0.99 and a specificity of 0.98 . Applying this threshold of $33 \mathrm{~s}$ hardly affected estimates of daily lying time, but improved estimates of frequency and mean duration of LB for individual cows. The importance of distinguishing short LB was demonstrated specifically for detection of calving. The 2-sensor approach, using sensor outputs on both hind legs as each other's validation, is a time-efficient method to validate LB records

Received March 10, 2015.

Accepted July 10, 2015.

${ }^{1}$ Corresponding author: akke.kok@wur.nl that can be applied to different sensors and husbandry conditions.

Key words: dairy cow, sensor, validation, lying behavior

\section{Technical Note}

Diseases, housing conditions, stocking density, temperature, and several other factors can cause changes in lying behavior (EFSA, 2009). Assessing lying behavior, therefore, can yield insight into the welfare of dairy cows. Lameness, for example, has been associated with an increase in total lying time (Ito et al., 2010). Furthermore, cows that had a difficult calf delivery alternated between lying and standing more often, resulting in a higher number of lying periods or lying bouts (LB) per day (Proudfoot et al., 2009).

Currently, lying behavior can be assessed using continuous observations from video recordings or data from sensors. Sensors have the potential to record lying behavior automatically, thus time efficiently. In addition, increasing use of activity sensors for estrus detection leads to an increasing on-farm presence of sensors that could also record lying behavior (Steeneveld and Hogeveen, 2015). Validation of the sensor output is necessary, however, to ensure that recorded data accurately reflect true behavior. Lying bout records have been validated against the gold standard of time-consuming behavioral observations to determine a threshold that retains true and discards false records (Trénel et al., 2009; Ledgerwood et al., 2010; Tolkamp et al., 2010; Mattachini et al., 2013). In other studies, however, thresholds are not used or not underpinned by scientific validations (Endres and Barberg, 2007; Ito et al., 2010; Blackie et al., 2011; Kokin et al., 2014).

IceTag sensors (IceRobotics, South Queensferry, UK), attached to one of the hind legs of a cow, have been used to record activity and lying behavior by several research groups (Endres and Barberg, 2007; Tolkamp et al., 2010; Blackie et al., 2011; Mattachini et al., 2013). Tolkamp et al. (2010) validated IceTag LB records against continuous observations of late- 
pregnant beef cows. They transformed data about the percentage of lying and standing per minute into lying episodes per second and defined a threshold of 4 min to discard false episodes. This threshold reduced the number of lying episodes with 62 to $88 \%$. Later, IceTag-data were produced per second and a threshold of $25 \mathrm{~s}$ was validated for dairy cows by Mattachini et al. (2013). The new IceManager software (2010) for the IceTag and similar IceQube sensor, which replaced IceTagAnalyser software, automatically creates a separate file with recorded LB. No LB record threshold has been formulated or validation performed for these LB records.

Thresholds for sensor output of lying behavior have been validated, so far, against behavioral observations (Ledgerwood et al., 2010; Tolkamp et al., 2010; Mattachini et al., 2013). Because behavioral observations take time, however, data sets to validate sensor output are often small (Trénel et al., 2009; Mattachini et al., 2013). Incidental short LB may not be observed frequently enough in such a data set to influence the threshold, while their detection will depend on it. Moreover, short LB could be highly relevant to detect as an indicator for acute discomfort or restlessness. Therefore, a larger data set would be more suitable to establish an optimal threshold to ensure that sensor data accurately reflect lying behavior.

We aimed to validate LB recorded by the IceQube sensor and to determine an optimal threshold to distinguish true from false LB records in lactating dairy cows. Moreover, to generate a larger data set that potentially includes more incidental short LB, we used a time-efficient novel method of validation that does not require behavioral observations. In addition, we specifically analyzed LB records of periparturient cows to illustrate the importance of detection of short LB.

\section{Experimental Setup and Data Collection}

In October and November 2014, data from 28 cows were obtained on the research farm Dairy Campus in Lelystad (the Netherlands). Cows were housed in free stalls with mattress and sawdust bedding and concrete slatted floors. They were milked twice daily and supplied with fixed amounts of concentrates and ad libitum roughage. Stage of lactation ranged from 20 to 133 DIM $(90 \pm 29$, mean $\pm \mathrm{SD})$. Parity ranged from 2 to $6(3.3 \pm 1.1)$. Cows were equipped with 2 IceQube sensors (IceRobotics) simultaneously. Sensors were attached to the left and the right hind leg of each cow, for a period of $7 \mathrm{~d}$, yielding paired records per cow. On November 7, 2014, the research farm was declared to have a Salmonella outbreak. Two weeks before that, fever and diarrhea were already observed in the herd.
It is unclear how many animals were infected. Diseases could affect total lying time, but are not expected to interfere with the recording and validation of LB data. Therefore, data from all cows were included. One cow died, resulting in only 1 complete day of recording.

To illustrate the importance of detecting short LB, we analyzed IceQube LB records around calving $(2 \mathrm{~d}$ before until $2 \mathrm{~d}$ after calving) from another 6 cows that calved on the research farm between August 2014 and July 2015.

IceReader (hardware; IceRobotics) was used to download IceQube data, and IceManager (software; IceRobotics) processed these data into LB records, with a start date, start time (hh:mm:ss) and duration (s). Per sensor, a file with LB records and a file with the number of LB recorded and lying time per day were produced. This output differs from earlier versions of IceTagAnalyser software, which only yielded the percentage of lying and standing recorded on a per-minute or per-second basis. The yet unvalidated LB records are referred to as raw LB records. Data were processed and analyzed in SAS (version 9.3, SAS Institute Inc., Cary, NC). The analysis at the LB record level included raw LB records collected from the $12 \mathrm{~h}$ following attachment of IceQube sensors, and from the subsequent 6 complete days. For analyses of lying time and LB frequency per day, only the 163 (27 cows $6 \mathrm{~d}$ and 1 cow $1 \mathrm{~d})$ completely recorded days were included.

Classification of raw LB records as true (actual lying) or false (recorded while standing) LB was based on 3 assumptions. First, all standing records (i.e., absence of a raw $\mathrm{LB}$ record between 2 consecutive raw LB records) were assumed to occur while standing (Tolkamp et al., 2010). Second, false LB records due to short leg movements from a vertical to a horizontal position could not occur in both hind legs simultaneously. Third, following from the first 2 assumptions, true LB only occurred if lying was recorded on both hind legs, thus when LB records of the paired sensors coincided. To classify the raw LB records, all were combined in one file and sorted by cow, start date, and start time. If the start date, start time, and duration of a raw LB record by the sensor on the right hind leg $(\mathbf{R})$ were identical to a raw LB record by the sensor on the left hind leg $(\mathbf{L})$ of the same cow, they were classified as a true LB record and assumed to correspond to a LB. However, start time and duration of coinciding raw $\mathrm{LB}$ records by $\mathrm{R}$ and L could differ slightly. This difference could be due to differences in leg movements when lying down and getting up, or could result from minor differences in the internal clock of both sensors. Therefore, the allowed difference in start time and duration was relaxed stepwise with $2 \mathrm{~s}$, until $14 \mathrm{~s}$, at which point all raw LB records that overlapped in time with raw LB records 
by the opposite sensor were classified as true. True LB records shorter than $30 \mathrm{~s}$ in duration were manually checked to verify overlap of the records in time.

\section{Data Analyses}

After raw LB records were classified as true or false $\mathrm{LB}$, the duration in seconds was $\log _{\mathrm{e}}$-transformed. We subsequently determined the accuracy, sensitivity, specificity, and positive and negative predictive value of thresholds for LB duration that were increased stepwise from 0 by $0.5 \log _{\mathrm{e}}$. Accuracy was defined as the sum of correctly discarded false LB records and correctly retained true $\mathrm{LB}$ records divided by the total amount of LB records (Weiss and Koepsell, 2014), sensitivity as the number of true LB retained divided by the total number of true LB, specificity as the number of false LB discarded divided by the total number of false LB, positive predictive value as the number of true LB retained divided by the total number of LB records retained, and negative predictive value as the amount of false LB discarded divided by the total amount of LB records discarded. Maximum accuracy was used to determine the most accurate threshold to distinguish true from false LB records.

To assess the effect of the determined threshold on estimates of lying time, number of LB per day, and LB duration (lying time per day/number of LB per day), these variables were expressed per day. This was done by summing LB records per cow per leg per start date of the LB record. The lying variables were computed using the following criteria: all raw LB records, only records longer than $33 \mathrm{~s}$, only records longer than 4 min, and only records classified as true LB. Lying variables were averaged per cow per sensor $(\mathrm{n}=56)$. We subsequently assessed the differences between variable estimates computed using no threshold, thresholds of $33 \mathrm{~s}$ and $4 \mathrm{~min}$, and variable values computed from true $\mathrm{LB}$, using descriptive statistics.

To determine the difference in number of LB per day around calving, repeated measures ANOVA and Bonferroni post-hoc tests were performed on LB records that exceeded the thresholds of $33 \mathrm{~s}$ and $4 \mathrm{~min}$ (PROC MIXED), in which measures of the subject cows ( $\mathrm{n}=$ 6 ) were compared between 5 subsequent days ( -2 to 2 d relative to calving).

\section{Results and Discussion}

On average, $12.1 \pm 3.3$ raw LB records were recorded per cow per day, which corresponded to a lying time of about $13 \mathrm{~h}$ per cow per day (13:02 $\pm 2: 18$, hh:mm). The difference in number of raw LB records per day between $\mathrm{R}$ and $\mathrm{L}$ on the same cow ranged from 0 to 7 .

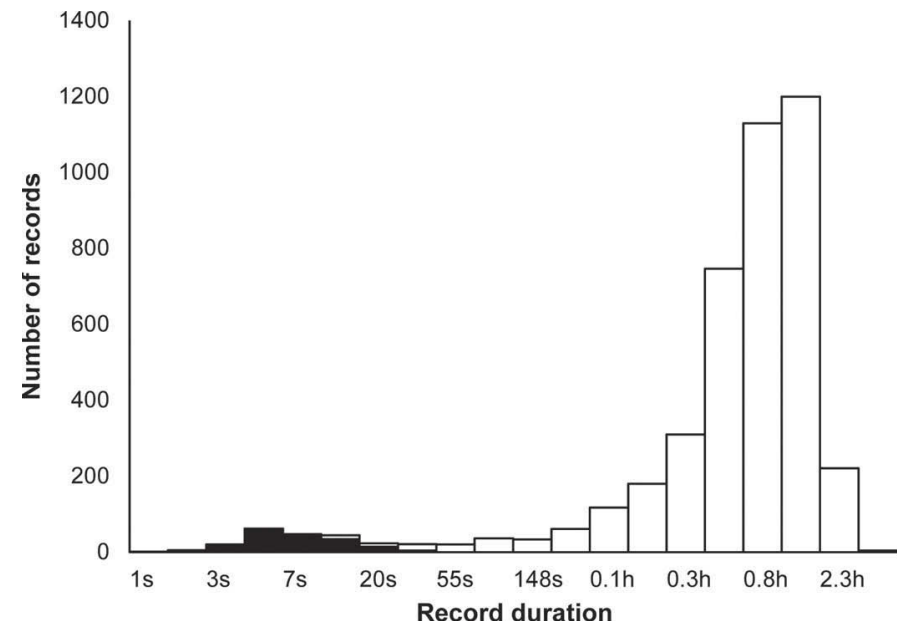

Figure 1. Duration and frequency of lying bout records. White bars represent true records; black bars represent false records.

The data set comprised 4,279 raw LB records. Only 307 of these raw LB records $(7.2 \%)$ had a duration shorter than 4 min, of which more than $60 \%$ (196) did not exceed $30 \mathrm{~s}$ (Figure 1).

Across cows, the number of true LB varied from 3.5 to 27 per day ( 8.5 to 16.5 when the 2 most extreme cows were excluded) and their duration ranged from $4 \mathrm{~s}$ to 4 $\mathrm{h}$ and $20 \mathrm{~min}$. The number of false LB records averaged 0.5 and ranged from 0 to 3.3 per day. Within cows, the difference in false LB records between hind legs ranged from 0 to 1.6 records per day. False LB records (4.7\%) included 177 short false LB records (from 1 to $50 \mathrm{~s}$ in duration), and 24 longer false LB records $(>20$ $\min )$. The longer false LB occurred in case one record from the sensor on one hind leg matched 2 subsequent records from the sensor on the opposite leg, separated by a nonlying period (of 5 to $13 \mathrm{~s}$ ). These $24 \mathrm{LB}$ records were not regarded as false, but were assumed to be 8 long true LB that were falsely interrupted by leg movements while lying.

As illustrated in Figure 1, the duration distributions of false and true LB overlapped. Therefore, no threshold could be defined that discarded all false and retained all true LB. A LB record threshold of $33 \mathrm{~s}$ (Table 1), however, yielded a maximum accuracy of 0.992 , a sensitivity of 0.993 , and a specificity of 0.977 . This threshold retained $2.5 \%$ more LB records than the threshold of 4 min indicated by Tolkamp et al. (2010). The retained data set still included $2.3 \%$ of the false LB records, which were outnumbered by true LB records of similar duration. In addition, the threshold of $33 \mathrm{~s}$ is close to the threshold of $25 \mathrm{~s}$ that was validated by Mattachini et al. (2013) and the sampling interval of 30 $\mathrm{s}$ in combination with a single-event data filter recommended by Ledgerwood et al. (2010). 
Table 1. False and true lying bout (LB) records retained (n) with increasing LB record thresholds and the associated accuracy (Acc), sensitivity (Sen), specificity (Sp), positive predictive value (PPV), and negative predictive value (NPV)

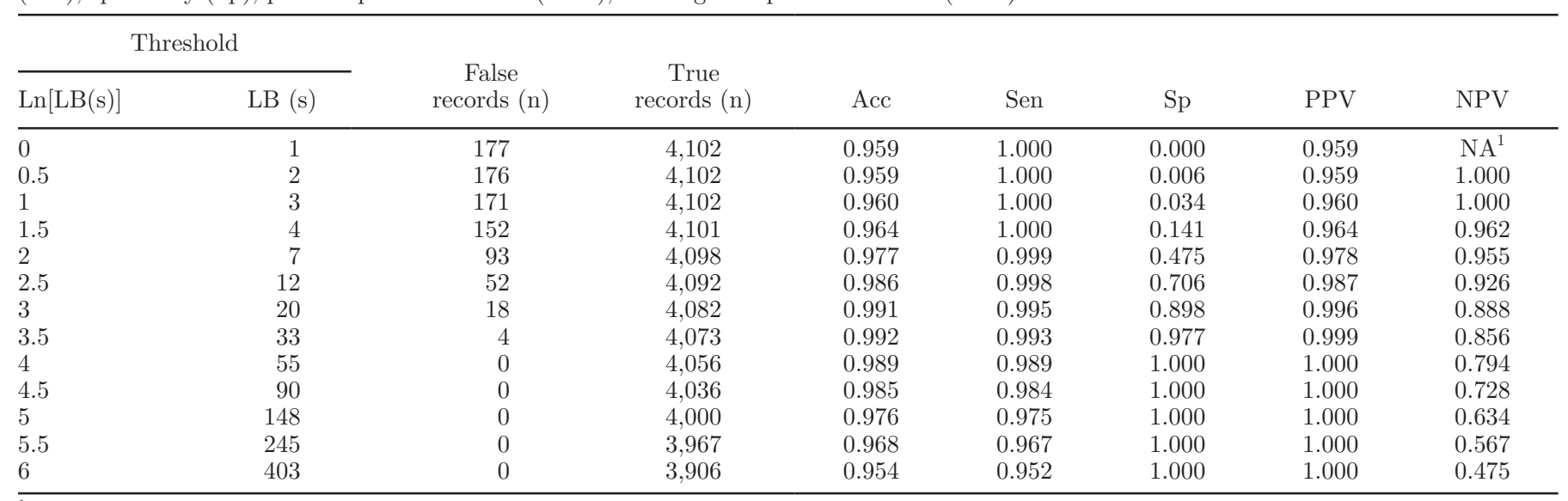

${ }^{1} \mathrm{NA}=$ not applicable.

Table 2 shows the effect of using different thresholds for LB record duration (i.e., no threshold, the determined threshold of $33 \mathrm{~s}$, and a threshold of 4 min as determined by Tolkamp et al., 2010) on estimates of daily lying time, frequency of $\mathrm{LB}$, and mean duration of LB per cow. The choice of threshold (i.e., no, $33 \mathrm{~s}$, or $4 \mathrm{~min}$ ) hardly affected estimates of daily lying time (i.e., the average difference between estimates using no threshold and using a threshold of $4 \mathrm{~min}$ was $1 \mathrm{~min}$ only). However, the choice of threshold did affect estimates of frequency and mean duration of LB.

The mean duration of LB was underestimated using no threshold, whereas it was overestimated by thresholds of $33 \mathrm{~s}$ and $4 \mathrm{~min}$. This was caused by the estimates of LB frequency, which were overestimated up to 3.3 LB per day using no threshold, whereas they were underestimated up to $0.5 \mathrm{LB}$ per day with a threshold of $33 \mathrm{~s}$ and $10 \mathrm{LB}$ per day with a threshold of $4 \mathrm{~min}$. The proportion of false LB (4.7\%, mean), however, varied from 0 to $49 \%$ between cows. As a result, the mean duration of LB for individual cows was underestimated up to 59 min when no LB record threshold was applied (see Table 3 for extreme cases). Both thresholds for LB record duration generally equalled or improved this estimate, with a maximum difference of 13 min between variable estimates and the true mean duration of $\mathrm{LB}$ for both thresholds. Overall, the LB record threshold of $33 \mathrm{~s}$ obtained the most accurate and least biased estimates for all lying variables.

The distribution of LB records presented here is different from the results of Tolkamp et al. (2010), in which more than $80 \%$ of the lying episodes was shorter than $4 \mathrm{~min}$. All these episodes were false in their experimental group of late-pregnant beef cows and resulted from leg movements such as scratching, from being displaced at the feeding rack and from teething problems such as loose wiring (B. J. Tolkamp and M. J. Haskell, Scotland's Rural College, Edinburgh, UK, personal communication). In our study, relatively few $(7.2 \%) \mathrm{LB}$ records had a duration shorter than $4 \mathrm{~min}$, and about half of those were assumed to be true LB. The great reduction in short LB records can probably be explained by the improved hardware, as reported by de Mol et al. (2013), and adjusted software that processes raw data into LB.

Whether the shortest LB records classified as true indeed correspond to true LB could be questioned, because our classification was not based on a golden standard but on the assumption that coinciding LB records only occur when the cow is lying. However,

Table 2. Difference between lying variable estimates per sensor per cow computed using no threshold, thresholds of $33 \mathrm{~s}$ and 4 min, and values computed from true lying bout $(\mathrm{LB})$ records

\begin{tabular}{|c|c|c|c|c|c|c|c|c|c|}
\hline Item & \multicolumn{3}{|c|}{$\Delta$ total lying time $(\min / \mathrm{d})$} & \multicolumn{3}{|c|}{$\Delta \mathrm{LB}$ duration (min/bout) } & \multicolumn{3}{|c|}{$\Delta \mathrm{LB}$ frequency $(\mathrm{n} / \mathrm{d})$} \\
\hline $\mathrm{SD}$ & 0 & 0 & 4 & 10 & 2 & 3 & 0.7 & 0.1 & 1.8 \\
\hline Minimum & 0 & 0 & -22 & -59 & -2 & 0 & 0.0 & -0.5 & -10.0 \\
\hline Maximum & 1 & 0 & 0 & 0 & 13 & 13 & 3.3 & 0.3 & 0.0 \\
\hline
\end{tabular}


Table 3. Estimates of mean lying bout (LB) duration and frequency for right (R) and left (L) hind legs of individual cows, computed from true LB records (true), all LB records (all), and LB records that exceed thresholds of $33 \mathrm{~s}$ and $4 \mathrm{~min}^{1}$

\begin{tabular}{|c|c|c|c|c|c|c|c|c|c|}
\hline \multirow[b]{2}{*}{ Cow } & \multirow[b]{2}{*}{ Leg } & \multicolumn{4}{|c|}{ LB duration (h/bout) } & \multicolumn{4}{|c|}{ LB frequency $(\mathrm{n} / \mathrm{d})$} \\
\hline & & True & All & $33 \mathrm{~s}$ & $4 \min$ & True & All & $33 \mathrm{~s}$ & $4 \mathrm{~min}$ \\
\hline 5177 & $\mathrm{~L}$ & 2.02 & 1.29 & 2.13 & 2.24 & 3.5 & 5.5 & 3.3 & 3.2 \\
\hline 5177 & $\mathrm{R}$ & 2.02 & 1.04 & 2.24 & 2.24 & 3.5 & 6.8 & 3.2 & 3.2 \\
\hline 6356 & $\mathrm{~L}$ & 1.43 & 1.18 & 1.43 & 1.46 & 9.3 & 11.3 & 9.3 & 9.2 \\
\hline 6356 & $\mathrm{R}$ & 1.43 & 1.32 & 1.43 & 1.46 & 9.3 & 10.2 & 9.3 & 9.2 \\
\hline 6459 & $\mathrm{~L}$ & 1.28 & 1.18 & 1.28 & 1.28 & 10.0 & 10.8 & 10.0 & 10.0 \\
\hline 6459 & $\mathrm{R}$ & 1.28 & 1.04 & 1.24 & 1.28 & 10.0 & 12.3 & 10.3 & 10.0 \\
\hline
\end{tabular}

${ }^{1}$ Only the data of 3 extreme individuals are presented for illustrative purposes.

short LB $(<4 \mathrm{~min})$ have regularly been observed in the herd. That such short true LB were not encountered in the study of Tolkamp et al. (2010) may be due to differences in lying behavior of late-pregnant beef cattle and lactating dairy cows.

Short LB could be informative for detection of restlessness, which could indicate issues such as (difficult) calving (Huzzey et al., 2005; Proudfoot et al., 2009) or other suboptimal conditions. In this study, the cow that died, for example, displayed restless lying behavior on the day before she died, including 10 (out of 27) LB between $33 \mathrm{~s}$ and $4 \mathrm{~min}$ in duration. For periparturient cows, the total number of LB per day using a threshold of 33 s peaked at $27.3 \pm 4.7$ (mean $\pm \mathrm{SD}$ ) on the day of calving, which was significantly higher than the number of LB on the $2 \mathrm{~d}$ before and after calving $(P<0.003$ for all contrasts of $\mathrm{d}-2,-1,1$, and 2 versus day of calving; Figure 2). Using a threshold of $4 \mathrm{~min}$, the number of LB numerically peaked at $22.0 \pm 3.3$ on the day of calving, but this was not significantly different from $\mathrm{d}$ -2 and -1 . The restlessness displayed by the cow that died and the periparturient cows would (at least partly) have been wrongly discarded by a threshold of $4 \mathrm{~min}$, indicating the potential importance of having a threshold as small as possible to retain accurate information on true lying behavior.

It was assumed in this study that the absence of LB records could only occur while standing, after findings of Tolkamp et al. (2010) that all short standing bout records in their study were true. The 8 interruptions of LB records in one hind leg while the sensor on the other leg recorded one LB, however, could be a false interruption of lying. If true standing, the cow would have stood up briefly while one hind leg remained in a horizontal position. False standing bouts may have been recorded due to movement of the leg while a cow was lying on her side. Cows have been observed to lie on their side and rest their hind leg on top of the cow in the adjacent cubicle in a diagonal position. Although this would violate our assumption, it remains a safe assumption to apply because these short interruptions occurred only incidentally.

The 2-sensor approach, using sensor outputs from opposite legs as each other's validation, is an easy and efficient method that can be applied to determine a threshold to distinguish true from false LB records. This in contrast to visual observations, which in case of continuous observations would be more accurate, but far more time consuming. It would be interesting to see if the currently proposed threshold of $33 \mathrm{~s}$ performs well for other sensors and under different husbandry conditions. Housing and management conditions, such as deep litter systems or grazing, influence lying behavior and movement of cows. A much larger overestimation of LB was reported for grazing than for cubicle-housed cows (27 vs. 1 LB in $4 \mathrm{~h}$ ), for example, but the duration of the false LB records was not reported (Rutter et al., 2014).

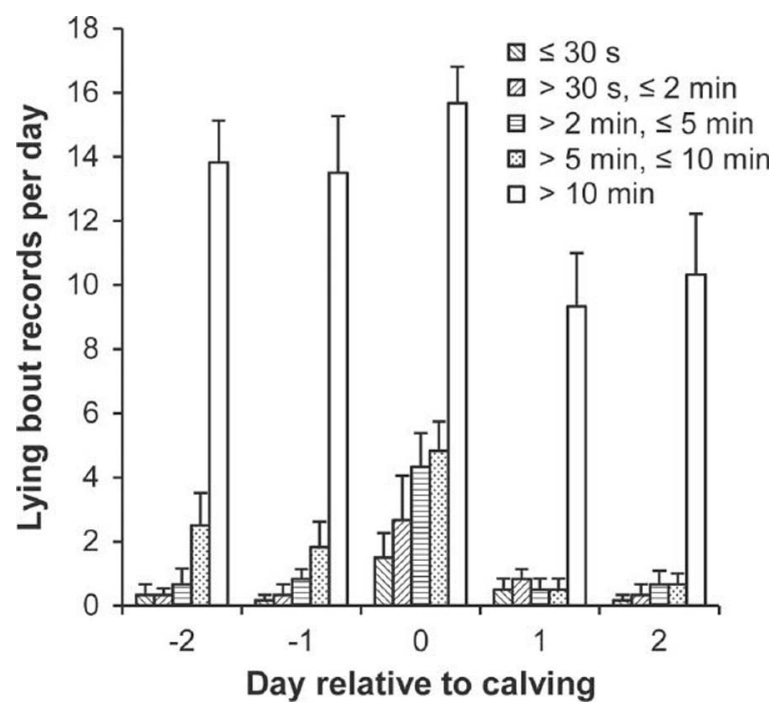

Figure 2. Mean (and SEM) number of lying bout records per cow $(\mathrm{n}=6)$ per day from $\mathrm{d}-2$ to 2 relative to calving, grouped into classes of duration. 
Next to validation, the usage of 2 sensors at a time provides a whole new scope of possibilities. It enables a classification of true and false LB records that is more accurate than a general LB threshold for all animals. Furthermore, it gives insight in the frequency and duration of false LB records. These false records could be indicative for frequency of grooming behavior, such as scratching and licking while standing on 3 legs, which may in turn reflect slipperiness of the floor (Platz et al., 2008) and mobility of the cows. Kokin et al. (2014) reported that the amount of LB records on the diseased hind leg of severely lame cows was more than twice the number recorded on the healthy leg. Although severe lameness may be detected visually, a change in false LB frequency could potentially be an early warning signal.

In conclusion, a minimum duration of LB records of $33 \mathrm{~s}$ was determined for lactating dairy cows to filter false records and accurately measure lying behavior using sensors. Applying this threshold is relevant for estimation of mean duration and frequency of LB of individual animals. Short LB records could be indicative of restlessness due to calving or illness. Moreover, using 2 identical sensors per cow is a time-efficient method for sensor validation and threshold determination, and can potentially yield information on cow health and welfare.

\section{ACKNOWLEDGMENTS}

The authors thank the staff of the Dairy Campus and B. de Schipper for support during the experiment, and C. G. van Reenen and J. T. N. van der Werf for sharing the IceManager software. This study is financed by DairyNL (Zuivel NL; organization of the Dutch dairy supply chain) and the Dutch Ministry of Economic Affairs (EZ), as part of the Sustainable Dairy Chain initiative (Duurzame Zuivelketen).

\section{REFERENCES}

Blackie, N., E. Bleach, J. Amory, and J. Scaife. 2011. Impact of lameness on gait characteristics and lying behaviour of zero grazed dairy cattle in early lactation. Appl. Anim. Behav. Sci. 129:67-73. http://dx.doi.org/10.1016/j.applanim.2010.10.006. de Mol, R. M., G. André, E. J. B. Bleumer, J. T. N. van der Werf, Y. de Haas, and C. G. van Reenen. 2013. Applicability of day-to-day variation in behavior for the automated detection of lameness in dairy cows. J. Dairy Sci. 96:3703-3712. http://dx.doi.org/http:// dx.doi.org/10.3168/jds.2012-6305.

EFSA (European Food Safety Authority). 2009. Scientific report of EFSA prepared by the Animal Health and Animal Welfare Unit on the effects of farming systems on dairy cow welfare and disease. Pages 1-284 Annex to the EFSA Journal, vol. 1143. EFSA, Parma, Italy.

Endres, M. I., and A. E. Barberg. 2007. Behavior of dairy cows in an alternative bedded-pack housing system. J. Dairy Sci. 90:41924200. http://dx.doi.org/10.3168/jds.2006-751.

Huzzey, J. M., M. A. G. von Keyserlingk, and D. M. Weary. 2005. Changes in feeding, drinking, and standing behavior of dairy cows during the transition period. J. Dairy Sci. 88:2454-2461. http:// dx.doi.org/10.3168/jds.S0022-0302(05)72923-4.

Ito, K., M. A. G. von Keyserlingk, S. J. Leblanc, and D. M. Weary. 2010. Lying behavior as an indicator of lameness in dairy cows. J. Dairy Sci. 93:3553-3560. http://dx.doi.org/10.3168/jds.2009-2951.

Kokin, E., J. Praks, I. Veermäe, V. Poikalainen, and M. Vallas. 2014. IceTag3D ${ }^{\mathrm{TM}}$ accelerometric device in cattle lameness detection. Agron. Res. 12:223-230.

Ledgerwood, D. N., C. Winckler, and C. B. Tucker. 2010. Evaluation of data loggers, sampling intervals, and editing techniques for measuring the lying behavior of dairy cattle. J. Dairy Sci. 93:51295139. http://dx.doi.org/10.3168/jds.2009-2945.

Mattachini, G., E. Riva, C. Bisaglia, J. C. A. M. Pompe, and G. Provolo. 2013. Methodology for quantifying the behavioral activity of dairy cows in freestall barns. J. Anim. Sci. 91:4899-4907. http:// dx.doi.org/10.2527/jas.2012-5554.

Platz, S., F. Ahrens, J. Bendel, H. H. D. Meyer, and M. H. Erhard. 2008. What happens with cow behavior when replacing concrete slatted floor by rubber coating: A case study. J. Dairy Sci. 91:9991004. http://dx.doi.org/10.3168/jds.2007-0584.

Proudfoot, K. L., J. M. Huzzey, and M. A. G. von Keyserlingk. 2009. The effect of dystocia on the dry matter intake and behavior of Holstein cows. J. Dairy Sci. 92:4937-4944. http://dx.doi. org/10.3168/jds.2009-2135.

Rutter, S. M., N. E. Atkins, and S. A. Birch. 2014. The accuracy of the automatic recording of lying behaviour is affected by whether dairy cows are inside or outside. Page 73 in 48th Congress of the International Society for Applied Ethology.

Steeneveld, W., and H. Hogeveen. 2015. Characterization of Dutch dairy farms using sensor systems for cow management. J. Dairy Sci. 98:709-717. http://dx.doi.org/10.3168/jds.2014-8595.

Tolkamp, B. J., M. J. Haskell, F. M. Langford, D. J. Roberts, and C. A. Morgan. 2010. Are cows more likely to lie down the longer they stand? Appl. Anim. Behav. Sci. 124:1-10. http://dx.doi. org/10.1016/j.applanim.2010.02.004.

Trénel, P., M. B. Jensen, E. L. Decker, and F. Skjøth. 2009. Technical note: Quantifying and characterizing behavior in dairy calves using the IceTag automatic recording device. J. Dairy Sci. 92:3397-3401. http://dx.doi.org/10.3168/jds.2009-2040.

Weiss, N. S., and T. D. Koepsell. 2014. Epidemiologic Methods: Studying the Occurrence of Illness. 2nd ed. Oxford University Press Inc., Oxford, UK. 